\title{
Dynamic Model of a 3-dof Asymmetric Parallel Mechanism
}

\author{
R. Z. H. Almeida* and T. A. Hess-Coelho*
}

Department of Mechatronics and Mechanical Systems Engineering, Escola Politécnica, University of São Paulo, Brazil

\begin{abstract}
Most research papers about parallel kinematic chain mechanisms investigate symmetric robot manipulators, in which all the limbs connecting the end-effector to the fixed based are composed by the same sequence of links and joints. Contrarily, in some manipulation tasks the velocity and stiffness requirements are anisotropic. In such cases, the asymmetric parallel kinematic chain mechanisms may offer advantages. This works' main objective is to present the inverse dynamic modeling of a 3-dof asymmetric parallel chain mechanism, conceived as a robot manipulator for pickand-place operations. The type of kinematic structure of the mechanism constrains the motion of the end-effector to only three translations. First, a brief kinematic modeling is carried out. Then, the inverse dynamic modeling is developed by employing the virtual work principle, considering two assumptions: lumped and distributed masses. Based on the model equations, a motion simulation is performed. The same motion is also analyzed by using the ADAMS computing environment to validate the model equations. After the simulation, the results demonstrated a very good agreement between the analyzed variables provided by the dynamic model and those generated by ADAMS. One can also notice that the input torques calculated by the lumped-mass model are quite close to the torques evaluated by the distributed mass model, and might be sufficient for the future development of a motion control law for the mechanism.
\end{abstract}

Keywords: Parallel robots, asymmetric mechanism, dynamic modeling, lumped/distributed masses, pick-and-place.

\section{INTRODUCTION}

The advantages of parallel kinematic chain mechanisms over serial kinematic chain ones are well known: high rigidity, lightness, fast dynamic response, high precision and high load capacity $[1,2]$. In some tasks, as pick and place operations, these advantages overcome the reduced workspace and the complexity of kinematic and dynamic modeling.

Some architectures, as those analyzed in [3-5], have reduced modeling complexity since their kinematic equations are linear and fully decoupled. However, due to the fact that these robots are overconstrained mechanisms, they require a very special care on manufacturing and assembly of their parts. Such requirements often demand more tight dimensional and geometrical tolerances, increasing their costs.

Most of the proposed parallel robot architectures present symmetric kinematic chains, while there are only few works dealing with asymmetric architectures [6,7]. This fact reflects the preference of researchers for a modular structure showing a behavior as close as possible to the isotropic. However, there are some applications in which the speed and rigidity requirements do not need to be the same in all the directions. For instance, the loading-unloading of goods in conveyor belts usually demand higher manipulator speeds in the transversal direction of transportation than in its main direction. As a result of this observation, a project concerning an asymmetric parallel manipulator robot for pick-andplace operations has been developed at the Department of

*Address correspondence to these authors at the Department of Mechatronics and Mechanical Systems Engineering, Escola Politécnica, University of São Paulo, Brazil; Tel: +551130915760; Fax: +551130915461; E-mails: rynaldoalmeida@usp.br, tarchess@usp.br
Mechatronics and Mechanical Systems of the Escola Politécnica of the University of São Paulo, Brazil [8].

As shown in Fig. (1), the kinematic structure of the proposed parallel mechanism is composed by three active limbs, connecting the moving platform with the fixed base. Two among them are RSS type, while the other is $\mathrm{PPaP}$, that is located in the central region of the mechanism. The letters $\mathrm{R}, \mathrm{P}$, and $\mathrm{S}$ stand for revolute, prismatic, and spherical joints, respectively. Here, Pa denotes a parallelogram subchain. The underlined letter means an active joint. The proposed kinematic structure $2 \underline{\mathrm{RSS}}+\underline{\mathrm{PPaP}}$ was conceived by apply-ing an alternative type synthesis $[9,10]$ procedure outlined in section 2 . The central limb $\mathrm{PPaP}$ was chosen in such a way that the moving platform only performs three translations.

This asymmetric and parallel architecture also presents an important feature: the kinematic equations are partially decoupled. Such feature is possible due to the fact that one of end-effector coordinates coincides with the displacement provided by the linear actuator coupled to the central limb.

The main goal of this paper is to develop the inverse dynamics model of the asymmetric parallel mechanism. Initially, in section 2, the kinematic modeling is derived. Then, in section 3, the dynamic model is developed for both lumped and distributed mass parameters. In section 4 , the results of the performed simulations are presented. Finally, in section 5 , the conclusions are enumerated.

\section{KINEMATIC DESIGN, MODELING AND ANA- LYSIS}

In order to generate a parallel mechanism able to position the end-effector in the $3 \mathrm{D}$-space, in such a way that it performs only three independent translations, an alternative type synthesis procedure proposed by Hess-Coelho [9] and cited 


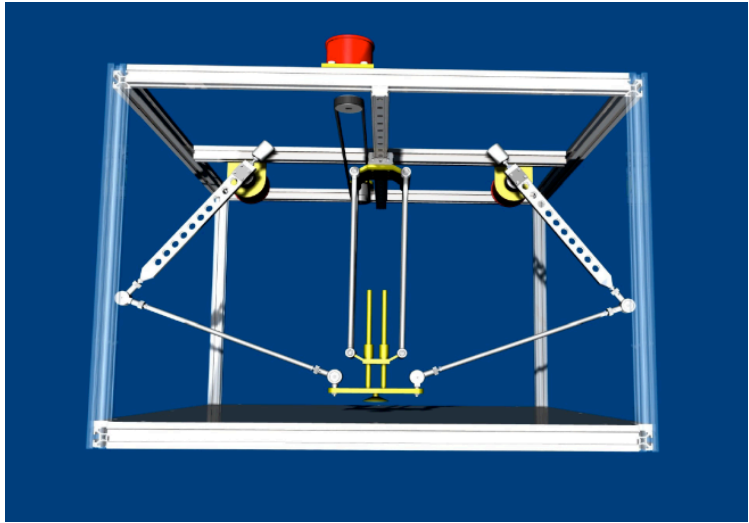

(a)

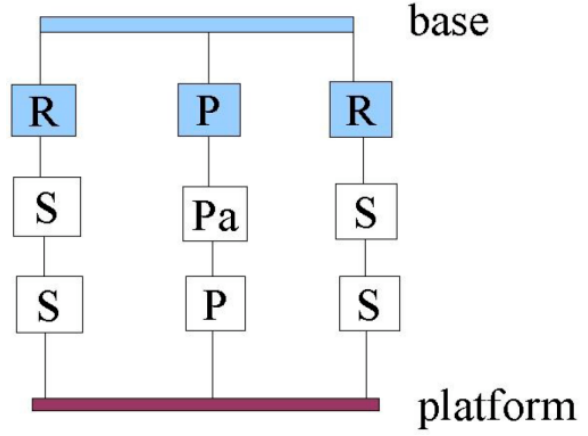

(b)

Fig. (1). Asymmetric parallel mechanism: (a) CAD model (b) graph representation.

in [11] is employed. Basically, the procedure has three steps: structural synthesis of the mechanism by the method of addition of passive limb; then, the elimination of one among the other active limbs; finally, setting active the constraining passive limb.

According to the first step, to constrain the end-effector motions, we choose the kinematic chain $\mathrm{PPaP}$ as the passive limb. Then, one feasible mechanism for the task is the 3 RSS $+\mathrm{PPaP}$. In fact, the peripheral limbs, whose connectivities equal 6 (discounting one passive degree of freedom [12] associated to the rotation about an axis through the centers of the spherical joints), do not restrict the tool movement. By applying the second step, we eliminate one active RSS limb. Finnally, we set active the constraining limb by coupling an actuator to drive the prismatic joint closest to the base.

The kinematic modeling of the parallel mechanism 2 $\underline{\mathrm{RSS}}+\mathrm{PPaP}$ is briefly derived here and a more detailed description can be found in [8]. The coordinates $q=\left[\begin{array}{lll}q_{1} & q_{2} & q_{3}\end{array}\right]^{T}$ correspond to the displacements provided by the actuators and the coordinates $\boldsymbol{x}=\left[\begin{array}{lll}x_{1} & x_{2} & x_{3}\end{array}\right]^{T}$ define the position of the end-effector, as it can be observed in Fig. (2), where $a_{\mathrm{i}}$ and $m_{\mathrm{i}}$ are the link lengths and masses and $\mathrm{M}$ is the load mass.

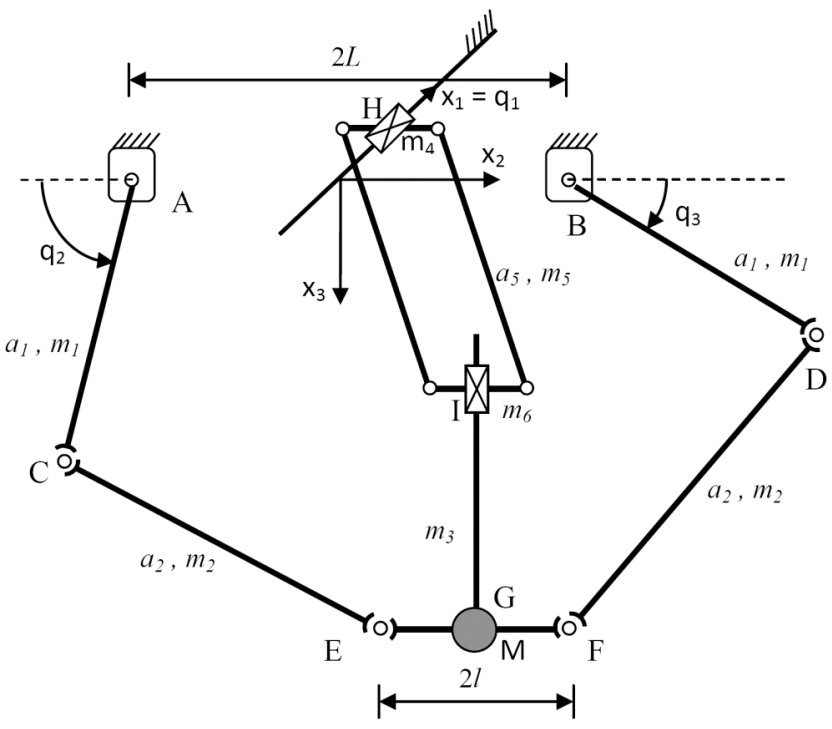

Fig. (2). Mechanism kinematic diagram.
The mathematical relations between the active joints coordinates and the end-effector coordinates are obtained from the property that the bar lengths $\mathrm{CE}$ and DF are constants. From the partial decoupling feature, the endeffector position along the $\mathrm{x}_{1}$ direction coincides with the active prismatic joint $\mathrm{q}_{1}$ coordinate. So, it can be stated that:

$\left\{\begin{array}{c}x_{1}-q_{1}=0 \\ x_{1}^{2}+\left(x_{2}-l+L+a_{1} \cos q_{2}\right)^{2}+\left(x_{3}-a_{1} \operatorname{sen} q_{2}\right)^{2}-a_{2}^{2}=0 \\ x_{1}^{2}+\left(x_{2}+l-L-a_{1} \cos q_{3}\right)^{2}+\left(x_{3}-a_{1} \operatorname{sen} q_{3}\right)^{2}-a_{2}^{2}=0\end{array}\right.$

Differentiating the eq.(1) with respect to time yields to eq.(2), a velocity equation written in a matrix form.

$J_{x} \dot{x}=J_{q} \dot{q}$

where:

$$
\begin{aligned}
& I_{x}=\left[\begin{array}{ccc}
1 & 0 & 0 \\
x_{1} & \left(x_{2}-l+L+a_{1} \cos q_{2}\right) & \left(x_{3}-a_{1} \operatorname{sen} q_{2}\right) \\
x_{1} & \left(x_{2}+l-L-a_{1} \cos q_{3}\right) & \left(x_{3}-a_{1} \operatorname{sen} q_{3}\right)
\end{array}\right] \\
& I_{q}=\left[\begin{array}{ccc}
J_{q 11} & 0 & 0 \\
0 & J_{q 22} & 0 \\
0 & 0 & J_{q 33}
\end{array}\right] \\
& I_{q 11}=1 \\
& I_{q 22}=\left(x_{2}-l+L+a_{1} \cos q_{2}\right)\left(a_{1} \operatorname{sen} q_{2}\right)+\left(x_{3}-a_{1} \operatorname{sen} q_{2}\right)\left(a_{1} \cos q_{2}\right) \\
& I_{q 33}=\left(x_{2}+l-L-a_{1} \cos q_{3}\right)\left(a_{1} \operatorname{sen} q_{3}\right)+\left(x_{3}-a_{1} \operatorname{sen} q_{3}\right)\left(a_{1} \cos q_{3}\right)
\end{aligned}
$$

The conditions for occurrence of singularities can be investigated by the inspection of the determinants of Jacobian matrices, $J_{x}$ and $J_{q}$ [12]. Fig. (3) shows two examples of singular configurations, where the PPaP limb was removed for clarity. When $\operatorname{det}\left(J_{q}\right)$ is null, the mechanism reaches the boundary of its workspace (Fig. 3a). On the other hand, when $\operatorname{det}\left(J_{x}\right)$ equals zero, the mechanism might become uncontrollable. In Fig. (3b) one can notice such configuration: the actuators at $\mathrm{A}$ and $\mathrm{B}$ cannot withstand vertical forces acting upon the end-effector. Fortunately, this condition will occur only if the parameter $a_{1}$ is larger than $a_{2}$.

The mechanism workspace was determined in [8] by a discretization method and its shape can be observed in Fig. (4). As expected, the workspace does not have a regular 


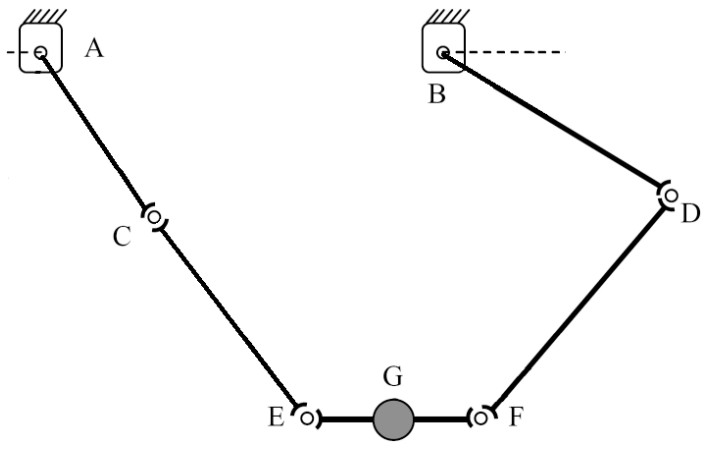

(a)

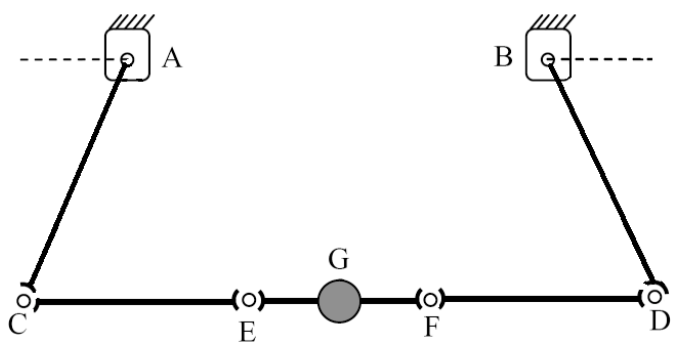

(b)

Fig. (3). Singularities: (a) workspace boundary (b) uncontrollable position.

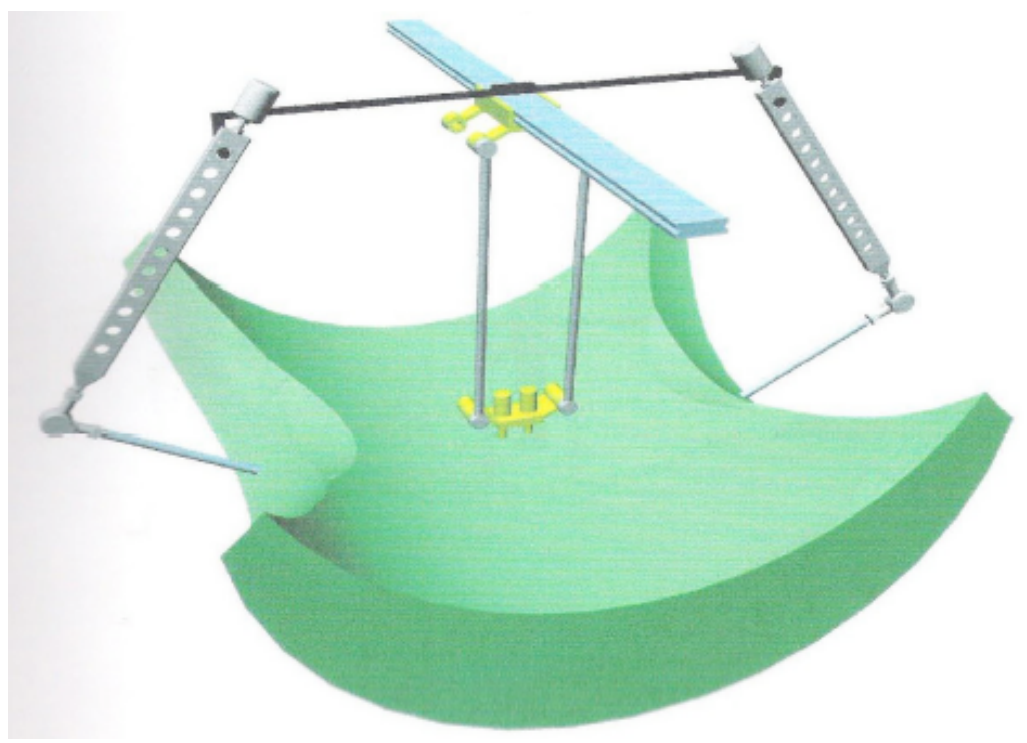

Fig. (4). Workspace shape.

shape but it may be improved by using adequate bar length ratio in each limb.

\section{DYNAMIC MODELING}

The mechanism dynamic modeling is developed here considering two different cases: lumped and distributed masses. In both cases, the bars are assumed to be rigid and the joints ideal (rigid, no friction and no clearance).

The virtual work principle is applied for the dynamic modeling due to the great easiness to build the equations, mainly because our focus is the inverse dynamic model of the mechanism, as it can be perceived in [13-15]. With this formulation approach, the input forces and torques appear as isolated terms from other variables in the derived equations. Moreover, the consideration of other effects such as joint friction, may be directly included in the model proposed here. Consequently, the dynamic model may be incrementally refined.

\subsection{Lumped Masses Modeling}

In this first case, we assume that the bars masses are lumped in their respective centers-of-mass. Hence, the mechanism itself is subject to the action of the inertia wrenches. In addition, the external forces and torques are due to the gravitational field and the actuators. Before applying the virtual work principle, it is important to realize that the virtual displacement and the center of mass acceleration of a rigid bar may be calculated from the virtual displacements and accelerations of its end points. Considering the homogeneous CE bar, for example, the eq. (3a-3b) may be used.

$\delta x_{C E}=\frac{\delta x_{C}+\delta x_{E}}{2}$

$\ddot{x}_{C E}=\frac{x_{C}+x_{E}}{2}$

where $\delta \boldsymbol{x}_{C E}$ and $\ddot{\boldsymbol{x}}_{C E}$ are the center-of-mass virtual displacement and acceleration of the CE bar.

These relations are very useful in the mechanism modeling because the virtual displacements and accelerations of points $\mathrm{E}$ and $\mathrm{F}$ equal to the virtual displacements and accelerations of the end-effector, respectively. On the other hand, the virtual displacements and accelerations of points $C$ and $D$ may be easily calculated from the movement of the active joints $\mathrm{q}_{2}$ and $\mathrm{q}_{3}$. Then, there is no need to find one jacobian matrix for each part of each mechanism limb. 
$\tau_{1} \delta q_{1}+\tau_{2} \delta q_{2}+\tau_{3} \delta q_{3}-m_{1} \frac{x_{C}}{2} \frac{\delta x_{C}}{2}-m_{2}\left(\frac{x_{C}+x_{E}}{2}\right)\left(\frac{\delta x_{C}+\delta x_{E}}{2}\right)-\left(m_{3}+M\right) \ddot{x} \delta \boldsymbol{x}-m_{1} \frac{x_{D}}{2} \frac{\delta x_{D}}{2}-$

$m_{2}\left(\frac{x_{D}+x_{F}}{2}\right)\left(\frac{\delta x_{D}+\delta x_{F}}{2}\right)-m_{4} \ddot{q}_{1} \delta q_{1}-2 m_{5}\left(\frac{x_{H}+x_{I}}{2}\right)\left(\frac{\delta x_{H}+\delta x_{I}}{2}\right)-m_{6} \ddot{x}_{l} \delta \boldsymbol{x}_{I}+m_{1} \boldsymbol{g} \frac{\delta x_{C}}{2}+m_{2} \boldsymbol{g}\left(\frac{\delta x_{C}+\delta x_{E}}{2}\right)+$

$\left(m_{3}+M\right) \boldsymbol{g} \delta \boldsymbol{x}+m_{1} \boldsymbol{g} \frac{\delta x_{D}}{2}+m_{2} \boldsymbol{g}\left(\frac{\delta x_{D}+\delta x_{F}}{2}\right)+m_{5} \boldsymbol{g} \frac{\delta x_{I}}{2}+m_{6} \boldsymbol{g} \delta \boldsymbol{x}_{I}=0$

Therefore, the sum of the virtual works of the actuators force and moments, inertia forces and gravitational forces must equals zero (eq. 4 ).

There $\tau=\left[\begin{array}{lll}\tau_{1} & \tau_{2} & \tau_{3}\end{array}\right]^{T}$ are the actuators force and moments and $g=\left[\begin{array}{lll}0 & 0 & 9,81\end{array}\right]^{T} \mathrm{~m} / \mathrm{s}^{2}$ is the gravity acceleration vector.

From the kinematics conditions, one can write the equations $5 \mathrm{a}-5 \mathrm{f}$.

$\delta \boldsymbol{x}=J_{x}^{-1} J_{q} \delta \boldsymbol{q}$

$\delta \boldsymbol{x}_{C}=\left[\begin{array}{lll}0 & a_{1} \sin q_{2} \delta q_{2} & a_{1} \cos q_{2} \delta q_{2}\end{array}\right]^{T}$

$\delta \boldsymbol{x}_{D}=\left[\begin{array}{lll}0 & -a_{1} \sin q_{3} \delta q_{3} & a_{1} \cos q_{3} \delta q_{3}\end{array}\right]^{T}$

$\delta \boldsymbol{x}_{E}=\delta \boldsymbol{x}_{F}=\delta \boldsymbol{x}$

$\delta \boldsymbol{x}_{H}=\left[\begin{array}{lll}\delta q_{1} & 0 & 0\end{array}\right]^{T}$

$\delta \boldsymbol{x}_{1}=\left[\begin{array}{lll}\delta q_{1} & \delta x_{2} & \frac{-x_{2}}{\sqrt{a_{5}^{2}-x_{2}^{2}}} \delta x_{2}\end{array}\right]^{T}$

Substituting the eq. (5a-5f) into eq.(4), we obtain eq. (6), in which only the actuated joints virtual displacements $\delta q_{1}$, $\delta q_{2}$ and $\delta q_{3}$ and their correspondent coefficients are present. $\left(\tau_{1}+f_{1}(\boldsymbol{q}, \dot{\boldsymbol{q}}, \ddot{\boldsymbol{q}})\right) \delta q_{1}+\left(\tau_{2}+f_{2}(\boldsymbol{q}, \dot{\boldsymbol{q}}, \ddot{\boldsymbol{q}})\right) \delta q_{2}+\left(\tau_{3}+f_{3}(\boldsymbol{q}, \dot{\boldsymbol{q}}, \ddot{\boldsymbol{q}})\right) \delta q_{3}=0$

Once the actuated joints virtual displacements in eq. (6) are independent, the algebraic expressions of force $\tau_{1}$ and the torques $\tau_{2}$ and $\tau_{3}$ can be obtained.

\subsection{Distributed Mass Modeling}

We intend here to extend the previous model by assuming the hypothesis of distributed mass along the links. In this case, it is sufficient to include the torques due to bars rotational inertia. The following terms must be added to the sum of virtual works for the bars with constant orientation rotation axis,

for bar $\mathrm{AC}$,

$$
-I_{A C \times 1} \ddot{q}_{2} \delta q_{2}
$$

for bar BD,

$$
-I_{B D \times 1} \ddot{q}_{3} \delta q_{3}
$$

for the parallelogram bars,

$$
-I_{H I x 1} \ddot{\theta}_{H} \delta \theta_{H}=-I_{H I x 1} \ddot{\theta}_{H} \frac{\delta x_{2}}{a_{5} \sin \theta_{H}}
$$

where $I_{A C x l}, I_{B D x l}$ and $I_{H I x l}$ are the AC, BD and parallelogram bars moments of inertia with respect to their principal axes that are parallel to the $\mathrm{x}_{1}$ direction and $\theta_{H}$ is the angle between the parallelogram bars and the horizontal direction, defined in the same way as $\mathrm{q}_{2}$.
For the CE and DF bars, of which the angular velocity vector has time varying orientation, it is necessary to include the following virtual work terms,

$$
\begin{aligned}
& \text { for bar CE: }\left(-\boldsymbol{I}_{C E} \dot{\boldsymbol{\omega}}_{C E}-\boldsymbol{\omega}_{C E} \wedge\left(\boldsymbol{I}_{C E} \boldsymbol{\omega}_{C E}\right)\right) \delta \boldsymbol{\Theta}_{C E} \\
& \text { for bar DF: }\left(-\boldsymbol{I}_{D F} \dot{\boldsymbol{\omega}}_{D F}-\boldsymbol{\omega}_{D F} \wedge\left(\boldsymbol{I}_{D F} \boldsymbol{\omega}_{D F}\right)\right) \delta \boldsymbol{\Theta}_{D F}
\end{aligned}
$$

where $\boldsymbol{I}_{C E}$ and $\boldsymbol{I}_{D F}$ are the CE and DF bars inertia matrices with respect to the global frame and $\omega_{C E}, \omega_{D F}, \delta \Theta_{C E}$ and $\delta \Theta_{D F}$ are the angular velocities and virtual displacements of the same bars. The inertia matrices $\boldsymbol{I}_{C E}$ and $\boldsymbol{I}_{D F}$ may be calculated from eq. (7-8) [12].

$I_{C E}=R_{C E} \bar{I}_{C E} R_{C E}^{T}$

$I_{D F}=R_{D F} \bar{I}_{D F} R_{D F}^{T}$

where $\bar{I}_{C E}$ and $\bar{I}_{D F}$ are the principal inertia matrices and $R_{C E}$ and $R_{D F}$ are the rotation matrices of the moving frames (body-fixed) with respect to the global frame.

For each bar, either $\mathrm{CE}$ or $\mathrm{DF}$, the angular velocity vector and its time derivative can be calculated from the velocities and accelerations vectors of the their end-points and from assuming that the angular velocity vector is always orthogonal to the longitudinal direction of the bar. In other words, there is no rotation along the longitudinal direction of the bar.

For the CE bar, for example, its angular velocity is obtained by:

$\boldsymbol{\omega}_{C E}=\left[\begin{array}{l}\left(x_{E 2}-x_{C 2}\right)\left(\dot{x}_{E 3}-\dot{x}_{C 3}\right)-\left(x_{E 3}-x_{C 3}\right)\left(\dot{x}_{E 2}-\dot{x}_{C 2}\right) \\ \left(x_{E 3}-x_{C 3}\right)\left(\dot{x}_{E 1}-\dot{x}_{C 1}\right)-\left(x_{E 1}-x_{C 1}\right)\left(\dot{x}_{E 3}-\dot{x}_{C 3}\right) \\ \left(x_{E 1}-x_{C 1}\right)\left(\dot{x}_{E 2}-\dot{x}_{C 2}\right)-\left(x_{E 2}-x_{C 2}\right)\left(\dot{x}_{E 1}-\dot{x}_{C 1}\right)\end{array}\right]$

Simplifying and rearranging the equation 9 , it may be written as follows

$\omega_{E C}=J_{\omega q} \dot{q}+J_{\omega x} \dot{x}$

where:

$I_{\omega q}=\frac{1}{a_{2}^{2}}\left[\begin{array}{ccc}0 & {\left[-\left(x_{2}-l+L\right) a_{1} \cos q_{2}+x_{3} a_{1} \sin q_{2}-a_{1}^{2}\right]} & 0 \\ 0 & a_{1} x_{1} \cos q_{2} & 0 \\ 0 & -a_{1} x_{1} \sin q_{2} & 0\end{array}\right]$

$I_{\omega x}=\frac{1}{a_{2}^{2}}\left[\begin{array}{ccc}0 & -\left(x_{3}-a_{1} \sin q_{2}\right) & x_{2}-l+L+a_{1} \cos q_{2} \\ x_{3}-a_{1} \sin q_{2} & 0 & -x_{1} \\ -\left(x_{2}-l+L+a_{1} \cos q_{2}\right) & x_{1} & 0\end{array}\right]$

Thus:

$\delta \boldsymbol{\Theta}_{\mathrm{CE}}=J_{\omega q} \delta \boldsymbol{q}+J_{\omega x} \delta \boldsymbol{x}=\left(J_{\omega q}+J_{\omega x} J_{x}^{-1} J_{q}\right) \delta \boldsymbol{q}$

Using equation 13, the torque due to bar $\mathrm{CE}$ rotational inertia equals to: 


$$
\left(-\boldsymbol{I}_{C E} \dot{\boldsymbol{\omega}}_{C E}-\boldsymbol{\omega}_{C E} \wedge\left(\boldsymbol{I}_{C E} \boldsymbol{\omega}_{C E}\right)\right)\left(\left(J_{\omega q}+J_{\omega x} J_{x}^{-1} J_{q}\right) \delta \boldsymbol{q}\right)
$$

that may be rearranged in the following form

$\left[\left(J_{\omega q}+J_{\omega x} J_{x}^{-1} J_{q}\right)^{T}\left(-I_{C E} \dot{\omega}_{C E}-\omega_{C E} \wedge\left(I_{C E} \omega_{C E}\right)\right)\right] \delta \boldsymbol{q}$

The same strategy can be applied to the bar DF. Finally, it is necessary to collect the terms dependent of $\delta q_{1}, \delta q_{2}$ and $\delta q_{3}$ and include them in eq. (6) to complete the dynamic model.

\section{SIMULATIONS}

Two different computing environments are employed to perform the mechanism motion simulation. The first environment corresponds to the kinematic and dynamic model, in accordance with the previous sections, implemented as Scilab [16] software subroutines. The ADAMS [17] software, a commercial package for multi-body analysis, is used as the second computing environment. Hence, the mathematical model and the correspondent implementation can be validated by comparing their results with those provided by ADAMS.

Notice that whereas the ADAMS results are obtained by numeric methods, the Scilab results are calculated from closed-form equations, which are suitable for future analysis, such as the modeling refinement and the implementation of a control law.

In order to make the end-effector reach the assorted positions, velocities and accelerations, we prescribe the input motions for the actuated joints, defined by sinusoidal functions with varied amplitudes and frequencies, as shown in eq. 16-18.

$q_{1}(t)=K_{1} \sin \left(\mathrm{k}_{1} \mathrm{t}\right)$

$q_{2}(t)=\pi / 4+K_{2} \sin \left(\mathrm{k}_{2} \mathrm{t}\right)$

$q_{3}(t)=\pi / 4+K_{3} \sin \left(\mathrm{k}_{3} \mathrm{t}\right)$

In the first simulation, the following numeric values were used for the required input motions and mechanism parts lengths and masses (Table $\mathbf{1}$ ).

Table 1. Simulations Parameters

\begin{tabular}{|c|c|c|}
\hline Input Motion & Bars Lengths [mm] & Bars and Load Masses [kg] \\
\hline \hline$K_{l}=100 \mathrm{~mm}$ & $L=200$ & $m_{1}=0,2$ \\
\hline$k_{l}=2$ & $l=100$ & $m_{2}=0,3$ \\
\hline$K_{2}=\pi / 12 \mathrm{rad}$ & $a_{1}=200$ & $m_{3}=0,45$ \\
\hline$k_{2}=5$ & $a_{2}=300$ & $m_{4}=0,1$ \\
\hline$K_{3}=\pi / 12 \mathrm{rad}$ & $a_{4}=100$ & $m_{5}=0,5$ \\
\hline$k_{3}=5$ & $a_{5}=250$ & $m_{6}=0,1$ \\
\hline & $a_{6}=100$ & $M=0 \mathrm{Kg}(\mathrm{no} \mathrm{load})$ \\
\hline
\end{tabular}

Fig. (5) shows the end-effector position as consequence of the active joints input position functions. The end-effector reaches $0,6 \mathrm{~m} / \mathrm{s}$ and $3,9 \mathrm{~m} / \mathrm{s}^{2}$. The simulations results for the lumped mass model with both computing environments can be found in Figs. (6-8). Each plot shows the necessary force or torque in each active joint.

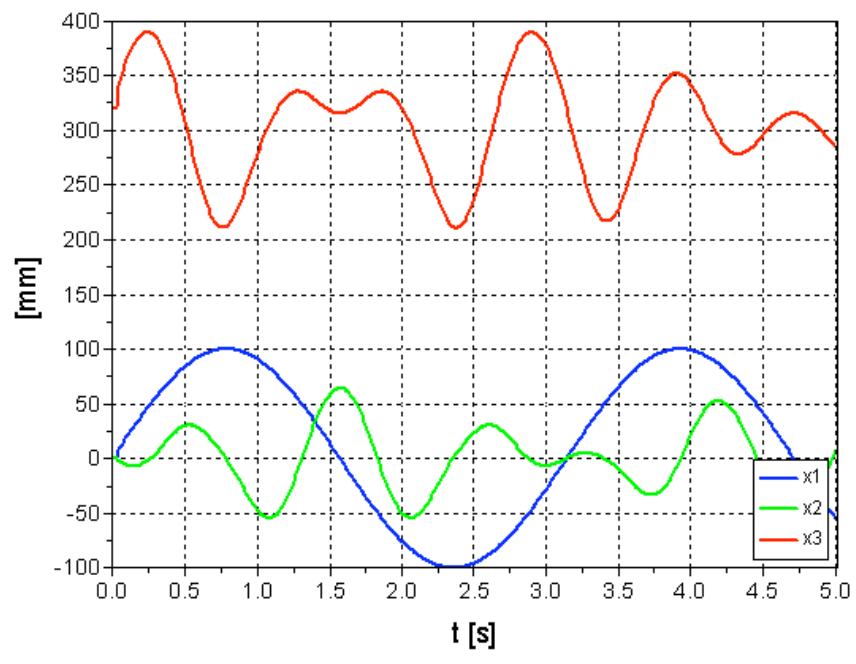

Fig. (5). End effector position.

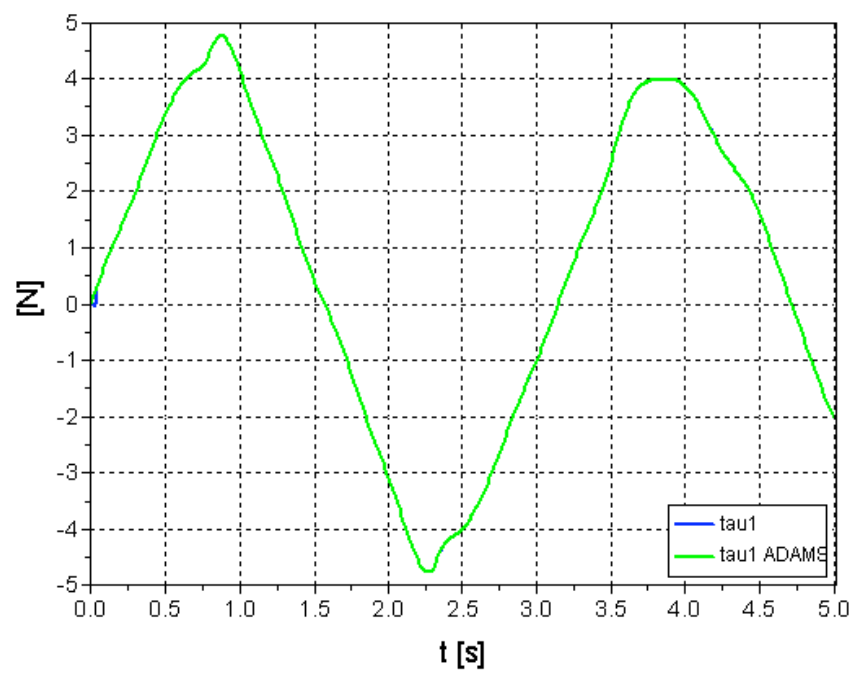

Fig. (6). Lumped masses: force $\tau_{1}$.

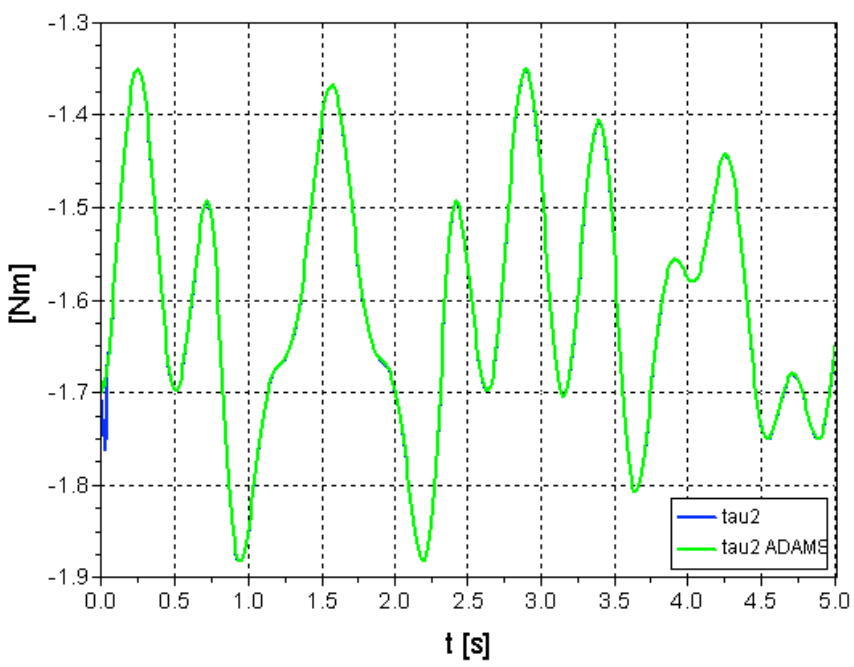

Fig. (7). Lumped masses: torque $\tau_{2}$. 


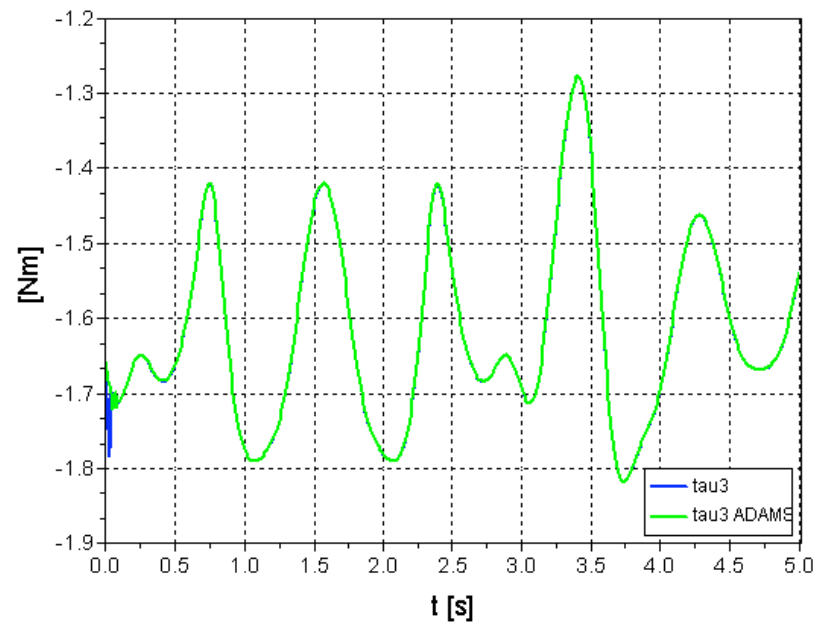

Fig. (8). Lumped masses: torque $\tau_{3}$.

One can observe that the result differences between the two computing environments are negligible. Considering the time interval $[0.1,5.0] \mathrm{s}$ the greatest relative error is smaller than $0.1 \%$. The initial instant was disregarded due to the large but not significant error caused by discontinuities in the velocity and acceleration functions in the movement start.

Figs. (9 to 11) show the necessary force and torques on the actuated joints for the distributed mass dynamic model.

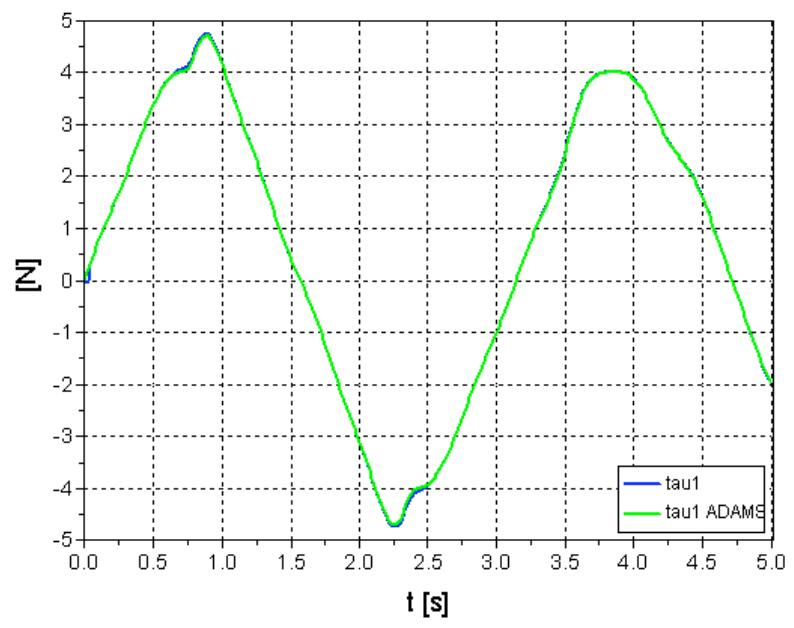

Fig. (9). Distributed masses: force $\tau_{1}$.

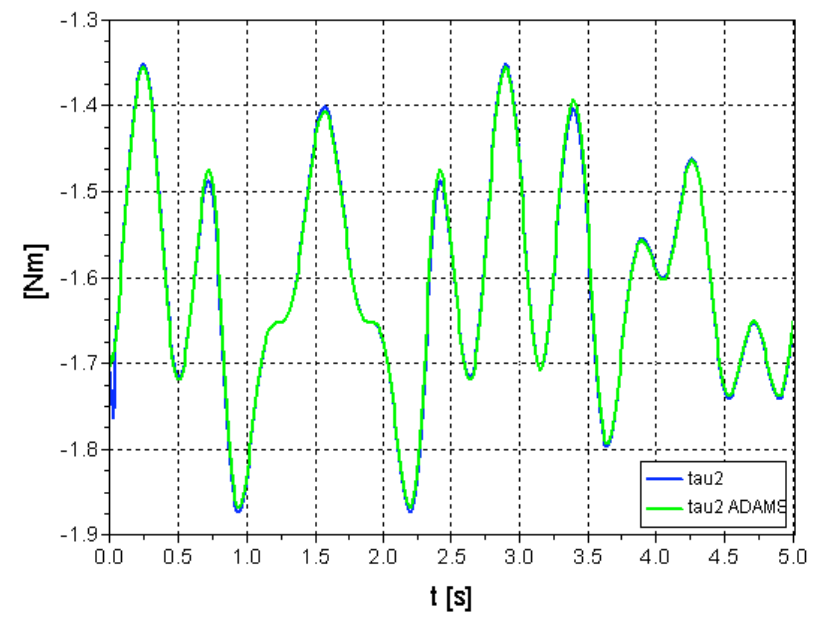

Fig. (10). Distributed masses: torque $\tau_{2}$.

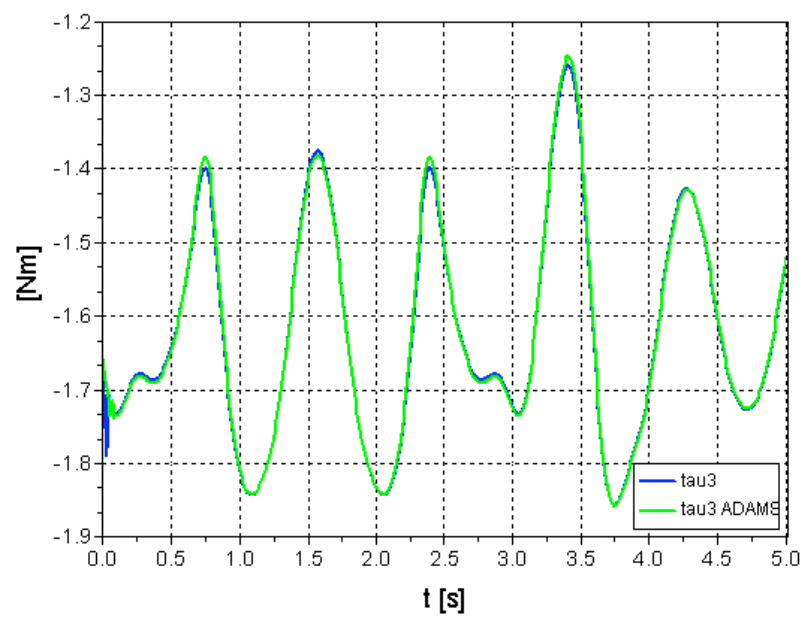

Fig. (11). Distributed masses: torque $\tau_{3}$.

The discrepancies between the results of the two simulation environments also remain small. The greatest relative error is smaller than $2 \%$. One can notice that the errors are greater in the peaks of the input torques associated with the coordinates $\mathrm{q}_{2}$ and $\mathrm{q}_{3}$, in which the second derivative of the torque functions with respect to time are significant.

In Figs. (12 and 13) the results provided by the Scilab environment of both cases of mass distribution assumptions are superposed. One can observe that the lumped mass model results are quite close to the model with distributed masses. The greatest relative errors are smaller than 5\% and show that the lumped mass model, simpler and less computationally demanding, probably would be sufficient for the development of a motion control law for the mechanism. We think that even better results would be reached if the position of the lumped masses would be moved from the bars original center of masses, as proposed by Codourey [18].

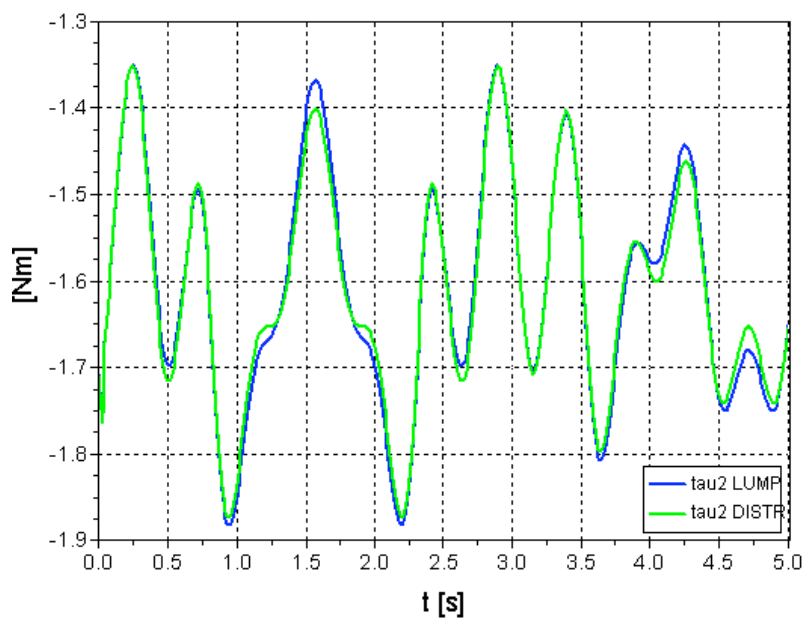

Fig. (12). Lumped/distributed masses: torque $\tau_{2}$.

Although the mathematical expressions were not simplified before the Scilab implementation, a computing cost comparison was accomplished. While the average distributed lumped mass model computing time was $703 \mu$ s per sample, the lumped mass model's was just $6,2 \mu$ s using an AMD Sempron $1,8 \mathrm{GHz}$ with $1 \mathrm{~GB}$ RAM. This demonstrates that a closed loop control based on this simpler model could employ a higher sample rate. However, higher errors might limit the operation to low performance movements. 


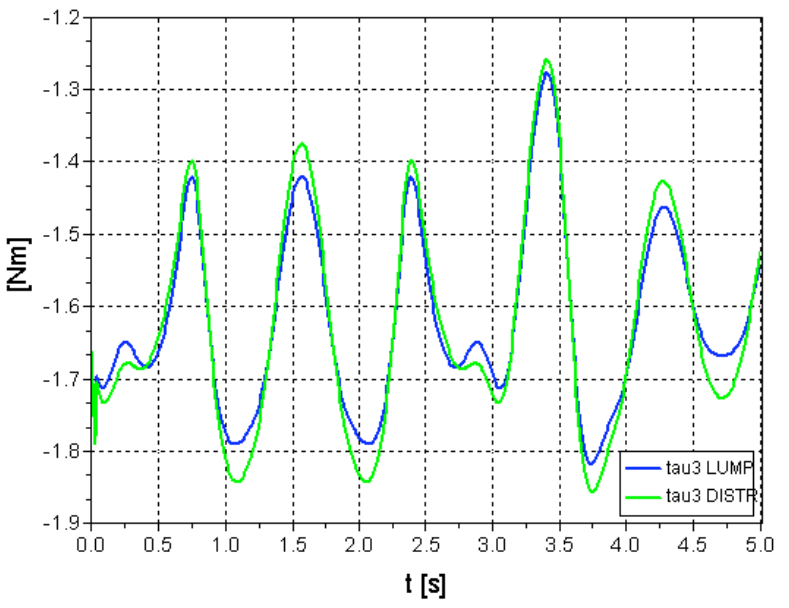

Fig. (13). Lumped/distributed masses: torque $\tau_{3}$.

In order to verify if the models remain close to each other even under higher performance movements, a second simulation was carried out with the same parameters of Table 1, except for $\mathrm{k}_{1}, \mathrm{k}_{2}$ and $\mathrm{k}_{3}$ that were changed to 10,25 and 35 , respectively. The simulation duration was reduced to one second. In this situation the end-effector reaches $2,9 \mathrm{~m} / \mathrm{s}$ and $97,5 \mathrm{~m} / \mathrm{s}^{2}$. The calculated required torques $\tau_{2}$ and $\tau_{3}$ may be observed in Figs. (14 and 15). Surprisingly, it can be perceived that the errors between the lumped and distributed model are still of the same magnitude.

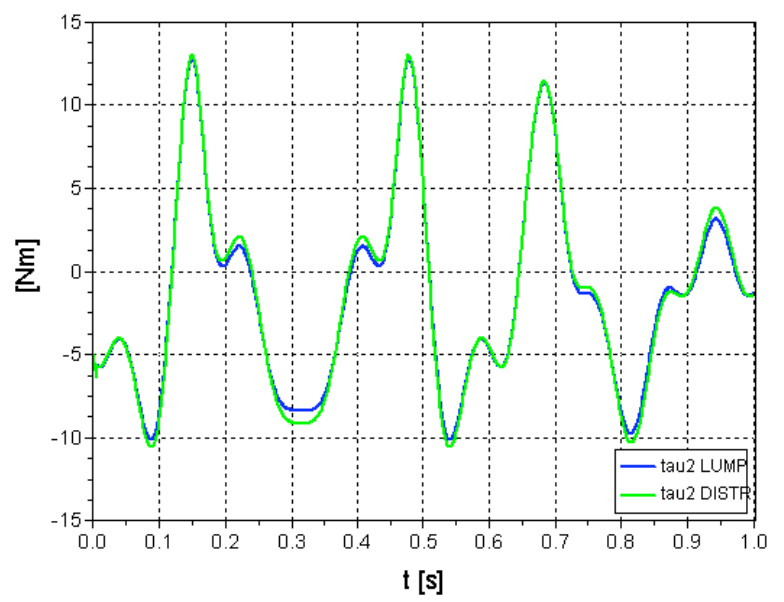

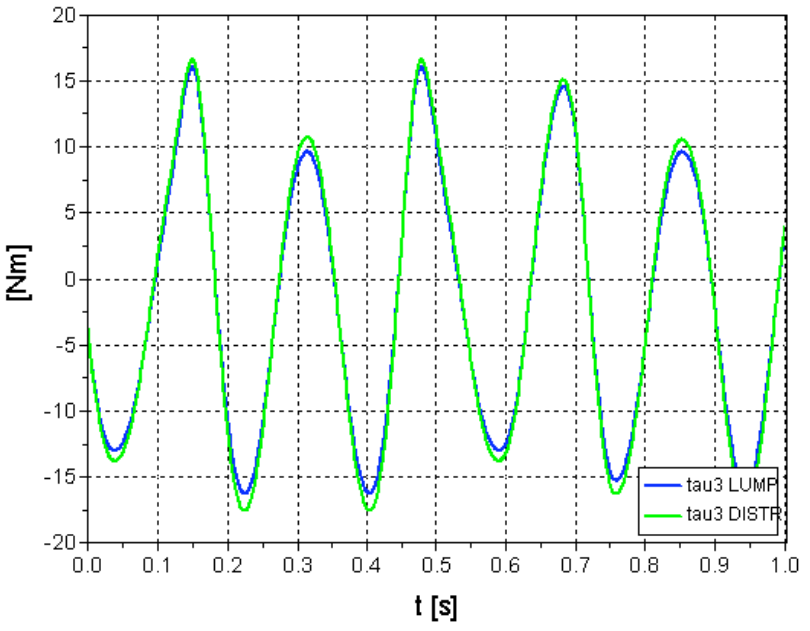

Fig. (15). Simulation 2: torque $\tau_{3}$.

\section{CONCLUSION}

Due to the fact that some industrial tasks have distinct motion requirements in each direction, asymmetric parallel mechanisms have a great potential to be more efficient and adequate for such applications. We developed here the dynamic model of the proposed asymmetric mechanism, which was successfully obtained for both the lumped mass and distributed mass assumptions.

The simulation results showed that the lumped mass model is a quite reasonable approximation of the more general distributed mass model and we think that would be enough for the development of motion control laws for the mechanism except under high performance movements. The virtual work principle demonstrated to be effective and efficient in the dynamic modeling and will allow the incremental refinement of the current model with future considerations as joint friction effects.

Fig. (14). Simulation 2: torque $\tau_{2}$.

\section{Appendix}

Expanded functions $f_{1}, f_{2}$ and $f_{3}$ of equation 6 .

$$
\begin{aligned}
& f_{1}=-K_{1}-\left(K_{4}+K_{5}\right) J_{x q 21}-K_{6} J_{x q 31} \\
& f_{2}=-K_{2}-\left(K_{4}+K_{5}\right) J_{x q 22}-K_{6} J_{x q 33} \\
& f_{3}=-K_{3}-\left(K_{4}+K_{5}\right) J_{x q 23}-K_{6} J_{x q 33}
\end{aligned}
$$

Where:

$$
\begin{gathered}
K_{1}=\left(\frac{m_{2}}{4}+m_{3}+m_{4}+m_{5}+m_{6}+M\right) \ddot{q}_{1} \\
K_{2}=\left(\left(m_{1}+m_{2}\right)\left(\frac{a_{1}^{2} \ddot{q}_{2}}{4}-\frac{g a_{1} \cos q_{2}}{2}\right)+\frac{m_{2} a_{1}}{4}\left(\ddot{x}_{2} \sin q_{2}+\ddot{x}_{3} \cos q_{2}\right)\right)
\end{gathered}
$$




$$
\begin{gathered}
K_{2}=\left(\left(m_{1}+m_{2}\right)\left(\frac{a_{1}^{2} \ddot{q}_{3}}{4}-\frac{g a_{1} \cos q_{3}}{2}\right)+\frac{m_{2} a_{1}}{4}\left(-\ddot{x}_{2} \sin q_{3}+\ddot{x}_{3} \cos q_{3}\right)\right) \\
K_{4}=\left(\frac{m_{2} a_{1}}{4}\left(\ddot{q}_{2} \sin q_{2}+\dot{q}_{2}^{2} \cos q_{2}-\ddot{q}_{3} \operatorname{sen} q_{3}-\dot{q}_{3}^{2} \cos q_{3}\right)+\left(\frac{m_{2}}{2}+m_{3}+\frac{m_{5}}{4}+m_{6}+M\right) \ddot{x}_{2}\right) \\
K_{5}=\left[\left(\frac{m_{5}}{4}+m_{6}\right)\left(\left(\dot{x}_{2}^{2}+x_{2} \ddot{x}_{2}\right)\left(a_{5}^{2}-x_{2}^{2}\right)^{-1 / 2}+\left(x_{2} \dot{x}_{2}\right)^{2}\left(a_{5}^{2}-x_{2}^{2}\right)^{-3 / 2}\right)+\left(\frac{m_{5}}{2}+m_{6}\right) g\right] x_{2}\left(a_{5}^{2}-x_{2}^{2}\right)^{-1 / 2} \\
K_{6}=\left(\frac{m_{2} a_{1}}{4}\left(\ddot{q}_{2} \cos q_{2}-\dot{q}_{2}^{2} \sin q_{2}+\ddot{q}_{3} \cos q_{3}-\dot{q}_{3}^{2} \sin q_{3}\right)+\left(\frac{m_{2}}{2}+m_{3}+M\right) \ddot{x}_{3}-\left(m_{2}+m_{3}+M\right) g\right) \\
l_{x q}=J_{x}^{-1} J_{q}
\end{gathered}
$$

\section{REFERENCES}

[1] J.P Merlet, "Still a long way to go on the road for parallel mechanisms", ASME DETC Confer., Montreal, Canada, 2002.

[2] M. Ceccarelli, Introduction to Robotic Manipulation, Dordrecht: Kluwer, 2004.

[3] H.S. Kim, and L.-W. Tsai, "Design optimization of a cartesian parallel manipulator", ASME DETC Confer., Montreal, Canada, 2002.

[4] C.M. Gosselin, X. Kong, S. Foucault, and I.A. Bonev, "A fullydecoupled 3-dof translational parallel mechanism", In Proc. 4th Chemnitz Parallel Kinematics Seminar, PKS2004, Verlag Wissenschaftliche Scripten, Reports from IWU, 2004, pp. 595-610.

[5] Di Gregorio, and V. Parenti-Castelli, "Design of 3-dof parallel manipulators based on dynamic performances", In Proc. of the 4th Chemnitz Parallel Kinematics Seminar, PKS2004, Verlag Wissenschaftliche Scripten, Reports from IWU, 2004, pp. 385-397.

[6] Q. Li, and Z. Huang, "Type synthesis of 5-DOF parallel manipulators" In Proc. of the 2003 IEEE Int. Conf. on Robotics and Automation Taipei, Taiwan, September 14-19: 1203-1208, 2003.

[7] T. Huang, M. Li, X.M. Zhao, J.P. Mei, D.G. Chetwynd, and S.J. $\mathrm{Hu}$, "Conceptual Design and Dimensional Synthesis for a 3-DOF Module of the TriVariant - A Novel 5-DOF Reconfigurable Hybrid Robot" IEEE Transactions on Robotics, vol. 21, No. 3, pp. 449456, 2005.

[8] V.D. Kumazawa, T. A. Hess-Coelho, D. Rinaudi, G. Carbone, and M. Ceccarelli, "Kinematic analysis and operation feasibility of a 3dof asymmetric parallel mechanism", In Proc. of the $20^{\text {th }}$ COBEM, Nov 15-20, Gramado, RS, Brazil, 2009.
[9] T.A. Hess-Coelho, "An alternative procedure for type synthesis of parallel mechanisms", In Proc. 12th IFToMM World Congress, Besançon, 2007.

[10] V.D. Kumazawa, Development of a Parallel Robot, Final Report, Escola Politecnica, Universidade de Sao Paulo, 2008 (available in portuguese).

[11] D.G. Gogu, Structural Synthesis of Parallel Robots, Parts 1 and 2, Kluwer, Dordrecht 2009.

[12] L.W. Tsai, Robot Analysis - The Mechanics of Serial and Parallel Manipulators. New York: John Wiley \& Sons, 1999.

[13] Y. Li, and Q. Xu, "Dynamic modeling and robust control of a 3PRC translational parallel kinematic machine", Robotics and Computer-Integrated Manufacturing, vol. 25, pp. 630-640, 2009.

[14] S. Staicu, "Dynamics analysis of the Star parallel manipulator", Robotics and Autonomous Systems, vol. 57, No. 11, pp. 1057-1064, 2009.

[15] J. Gallardo-Alvarado, C. Aguilar-Najera, L. Casique-Rosas, J. Rico-Martinez, M. Nazrul Islam, "Kinematics and dynamics of 2(3-RPS) manipulators by means of screw theory and the principle of virtual work", Mechanism and Machine Theory, vol. 43, No. 10, pp. 1281-1294, 2008.

[16] Scilab Consortium, Scilab manual, available online at http://www. scilab.org, accessed on June 9, 2010.

[17] MSC Software Corporation, MD Adams Basic Full Simulation. ADM 701 Course notes, 2007.

[18] A. Codourey, "Dynamic modeling and mass matrix evaluation of the DELTA parallel robot for axes decoupling control". In Proc. of IEEE/RSJ International Conference on Intelligent Robots and Systems Osaka, pp. 1211-1218, 1996.

Received: March 09, 2010

(C) Almeida and Hess-Coelho; Licensee Bentham Open.

This is an open access article licensed under the terms of the Creative Commons Attribution Non-Commercial License (http: //creativecommons.org/licenses/by$\mathrm{nc} / 3.0 /$ ), which permits unrestricted, non-commercial use, distribution and reproduction in any medium, provided the work is properly cited. 